\title{
Correlations between histological type, clinical behaviour, and prognosis in thymoma
}

\author{
COSTANTE RICCI, ERINO A RENDINA, EDOARDO O PESCARMONA, \\ FEDERICO VENUTA, ROCCO DI TOLLA, LUIGI P RUCO, CARLO D BARONI
}

From the Department of Thoracic Surgery and the Second Department of Pathological Anatomy, University of Rome "La Sapienza," Rome, Italy

ABSTRACT Seventy four cases of thymoma were reclassified into three histological categoriescortical (30), medullary (9), and mixed (34) (the remaining patient had an intrathymic thymoma)for an investigation of the relation between histological type, clinical behaviour, and long term prognosis. There were significant differences between the histological types in the frequency of myasthenia gravis and of the different tumour stages, the mean age of the patients, and prognosis. Myasthenia gravis occurred more commonly in patients with cortical (33\%) and mixed thymoma $(35 \%)$ than in patients with medullary thymoma (11\%). Five, 10, 15, and 20 year actuarial survival was $100 \%$ for medullary thymoma; $85 \%, 76 \%, 65 \%$ and $65 \%$ respectively for mixed thymoma; and $52 \%, 45 \%, 45 \%$, and $45 \%$ for cortical thymoma. Medullary thymoma is a benign tumour arising late in life and there was no mortality in this series after surgery alone. Cortical thymoma usually presented in middle age and must be regarded as malignant; mortality was $50 \%$ at five years despite a multidisciplinary approach, with surgery and postoperative radiotherapy in all patients and chemotherapy in selected cases. Mixed thymoma had a better prognosis than cortical thymoma, but must be regarded as potentially malignant. One third of the total patients had died by 10 years despite radical tumour resection.

\section{Introduction}

Thymomas, defined as thymic epithelial neoplasms with minimal or no cytological atypia, ${ }^{1}$ are charasterised by a remarkable morphological heterogeneity ${ }^{2}$ and variable clinical behaviour. ${ }^{34}$ Histological classifications in current use are based purely on morphology ${ }^{245}$ and have little prognostic or therapeutic value. In consequence, treatment is at present based solely on the surgical stage.

The classification recently proposed by Marino and Müller-Hermelink ${ }^{6}$ takes both morphology and histogenesis into account, and provides a new approach to management. In a preliminary investigation $^{7}$ we found that the classification correlated well with the surgical stage of the tumour. In the present study we have analysed the relation between clinical features, long term prognosis, and histological type in a larger group of patients.

Address for reprint requests: Dr E A Rendina, Cattedra di Chirurgia Toracica, II Clinica Chirurgica, Università di Roma "La Sapienza," 00161 Roma, Italy.

Accepted 13 March 1989

\section{Methods}

From January 1965 to December 1987120 patients with thymoma were treated surgically in the department of surgery of the University of Rome. In 38 cases insufficient pathological material was available for review, and eight patients were lost to follow up. The remaining 74 cases form the basis of this investigation.

Sections $4 \mu \mathrm{m}$ thick were stained for conventional histological examination with haematoxylin and eosin and the Giemsa, periodic acid-Schiff, and Gomori techniques, and examined by the same pathologists (CDB, EOP, LPR), all of whom have specific experience in the subject. Invasiveness was graded according to the criteria of Masaoka. ${ }^{8}$ Symptoms and signs, paraneoplastic syndromes, completeness of resection, and follow up were related to histological type. Survival was calculated for 73 patients by the actuarial method -one patient with intrathymic thymoma was excluded. Student's $t$ test and $\chi^{2}$ test were used for statistical analysis.

Histological ClASSIFICATION

In this study a classification based on that of Marino 


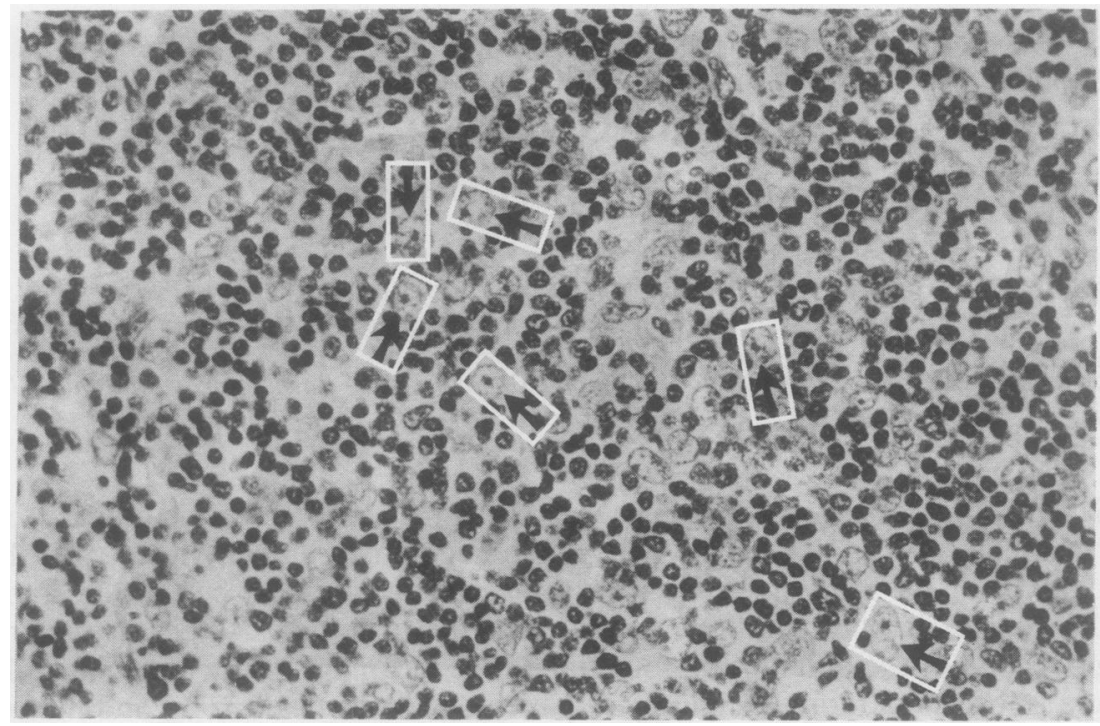

Fig 1 Cortical thymoma: epithelial cells of the cortical type with prominent nucleoli (arrows) and scattered lymphocytes.

and Müller-Hermelink ${ }^{6}$ was used, but categorising thymomas into only three types-cortical, mixed, and medullary.

By this classification cortical thymomas are defined as tumours composed mainly of medium sized to large epithelial cells, with round or oval nuclei, finely dispersed chromatin, prominent central nucleoli, and an ill defined cytoplasm (fig 1). Lymphocytes are usually abundant, often with a blastic appearance. There is frequently a "starry sky" pattern, focal medullary differentiation with Hassal's corpuscles, and perivascular epithelial cell palisading.

Medullary thymomas are composed of small to medium sized cells with irregular, often spindle shaped nuclei devoid of nucleoli (fig 2). Lymphocytes, generally present in small numbers, are of the mature

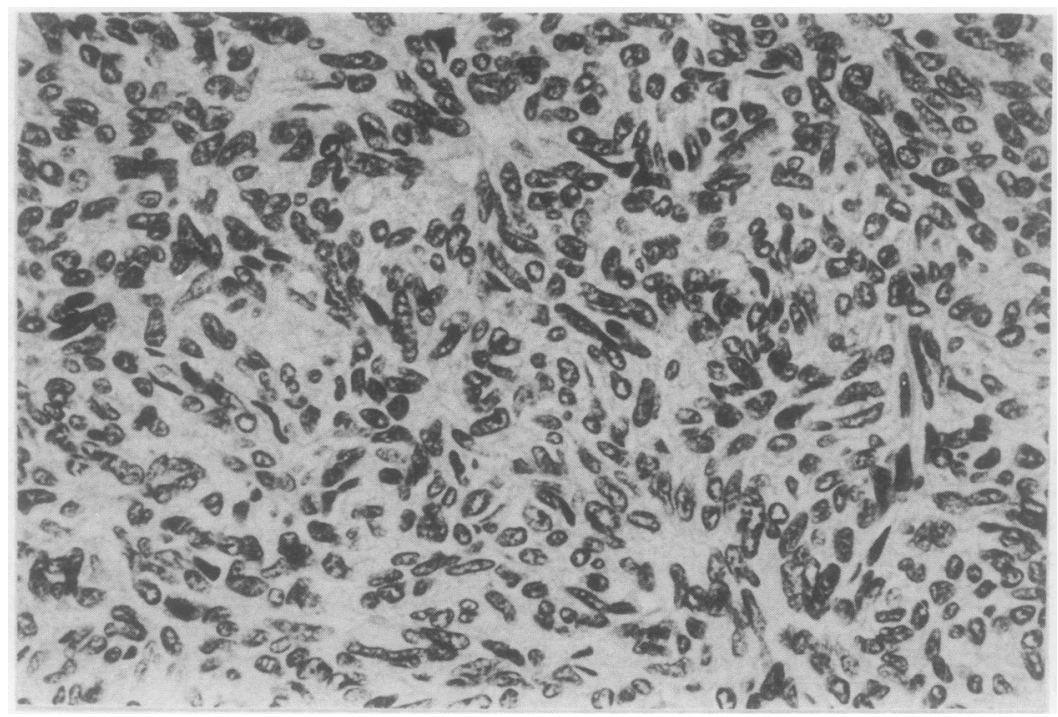

Fig 2 Medullary thymoma: spindle shaped epithelial cells of medullary type with a scanty lymphoid component. 


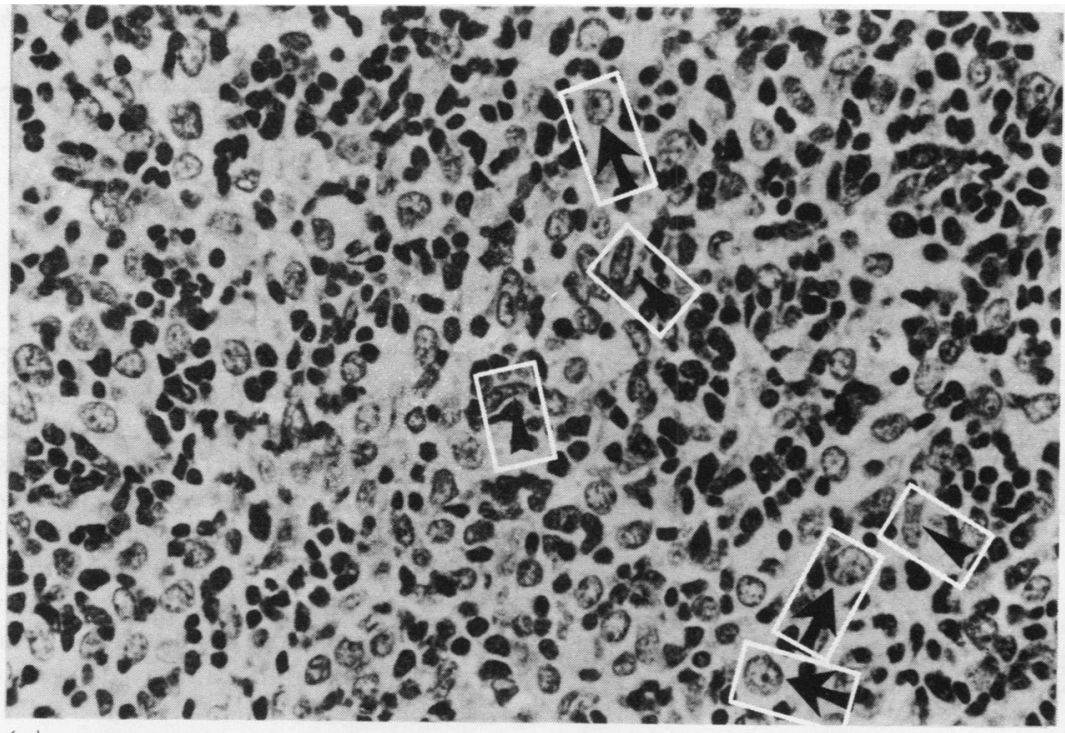

(a)

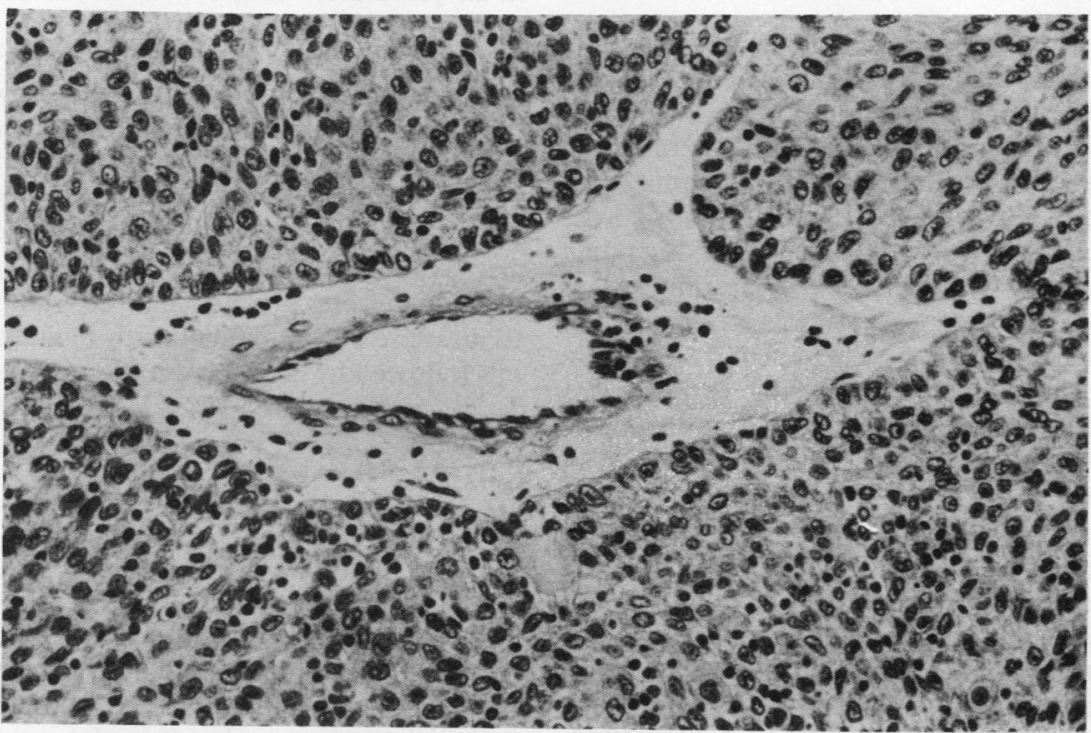

(b)

Fig 3 Mixed thymoma: (a) cortical (arrows) and medullary (arrowheads) type epithelial cells with a few lymphocytes; (b) dilated perivascular spaces, a typical feature of mixed thymoma.

thymocytic type. Epithelial lined cysts are often present, and a storiform pattern is sometimes observed.

In mixed thymomas epithelial components of cortical and medullary type are both present, intermingled with a variable number of lymphocytes (fig
$3 a$ ). Dilated and sometimes hyaline perivascular spaces are frequent (fig $3 b$ ). Mixed thymomas with a clear predominance of cortical or medullary differentiation were assigned to the cortical and the medullary group respectively.

Intrathymic thymoma, composed of abnormal 
Table 1 Relation between histological type of thymoma, myasthenia gravis, and surgical stage

\begin{tabular}{|c|c|c|c|c|c|c|c|c|}
\hline \multirow[b]{2}{*}{ Histological type (n) } & \multicolumn{4}{|c|}{ Stage $e^{*}$} & & \multicolumn{3}{|c|}{$\begin{array}{l}\text { Myasthenia } \\
\text { gravist }\end{array}$} \\
\hline & $I$ & II & III & $I V$ & & + & - & $\%$ \\
\hline CT (30) & - & 6 & 19 & 5 & & 10 & 20 & 33 \\
\hline MxT (34) & 12 & 15 & 6 & 1 & & 12 & 22 & 35 \\
\hline MT (9) & 8 & 1 & - & - & & 1 & 8 & 11 \\
\hline Intrathymic (1) & 1 & - & - & - & & i & - & 100 \\
\hline Total & 21 & 22 & 25 & 6 & - & 24 & 50 & \\
\hline
\end{tabular}

*Distribution differs significantly between the groups $\left(\chi^{2}=41 \cdot 684\right.$, $\mathrm{p}<0.001$ ).

†Frequency differs significantly between the groups $\left(\chi^{2}=158.78\right.$, $\mathrm{p}<0.001$ ).

CT-cortical thymoma; MxT-mixed thymoma; MT-medullary thymoma.

microscopic islands of epithelial cells surrounded by normal thymic parenchyma, appeared only once in the series and will not be considered further.

\section{Results}

Thirty six patients were male and 38 female, their ages ranging from 14 to 82 years, with a mean of 48 years. Thirty had a cortical thymoma and nine a medullary thymoma, and in 34 cases the tumour was of mixed type. The patients with medullary thymoma were older (mean age 69 years $-p<0.001$ ) than patients with mixed thymoma (47 years) and cortical thymoma (43 years). Sixteen patients were symptom free at presentation. Myasthenia gravis was present in 24 $(32 \%)$, and its incidence was significantly lower in patients with medullary thymoma (table 1). There was a significant difference in stage at presentation among the three types, most of the cortical thymomas being at stage III and most of the medullary and mixed tumours at stage I or II (table 1). Three patients with cortical thymoma had superior vena caval occlusion and one had erythroid hypoplasia.

Sixty nine patients had undergone surgery on one occasion (preceded by mediastinoscopy in five instances); four had had two operations and one three operations. Surgical procedures are detailed in table 2. In one patient with extension of tumour to the diaphragm and in one with pleural and diaphragmatic metastases resection was completed by thoracotomy through the sixth intercostal space after the bulk of the tumour had been removed via a median sternotomy. Reconstruction of the superior vena cava by a synthetic prosthesis was necessary in one patient with cortical thymoma, and an upper lobectomy was carried out because of direct tumour infiltration in one case of mixed thymoma. Major postoperative complications occurred in five patients and two died, giving an operative mortality of $2 \cdot 7 \%$. Radiotherapy
Table 2 Surgical procedures performed in the 74 patients with thymoma

\begin{tabular}{lrc}
\hline & \multicolumn{3}{c}{ No (\%) of procedures } \\
\hline Median sternotomy & 51 & $(59)$ \\
Thoracotomy & 20 & $(23)$ \\
Sternal split & 7 & $(9)$ \\
Mediastinoscopy & 5 & $(6)$ \\
Sternotomy and thoracotomy & 2 & $(2)$ \\
Anterior mediastinotomy & 1 & $(1)$ \\
& 86 & $(100)$ \\
\hline
\end{tabular}

was given routinely to patients with stage III and IV disease, and chemotherapy was combined with radiotherapy in three patients with stage 4 disease.

Radical resection was possible in 60 of the 74 cases; palliative resection was performed in six and biopsy alone in eight. All patients with medullary thymomas underwent radical resection, whereas in 10 of the 30 patients with cortical thymoma and in four of 34 with mixed thymoma only palliative surgery or biopsy was possible.

All patients with medullary thymoma are alive two to 22 years after surgery; for those with mixed and cortical thymoma the prognosis was significantly worse, with actuarial survival rates of $65 \%$ and $45 \%$ at 20 years (table 3 and fig 4). Six patients with stage I or II cortical or mixed thymoma died of the disease. All of the patients with relapsing disease that required further surgery had a cortical thymoma; three had stage III or IV tumours and had undergone radiotherapy after the first operation. In two patients stage II lesions were present and no radiotherapy was administered. One patient with medullary thymoma was followed by computed tomography for five years (fig 5); the tumour remained localised throughout this period.

\section{Discussion}

Neoplasms of the thymus differ in histogenesis, and epithelial, lymphoid, neuroendocrine, germ cell, and mesenchymal tumours have all been described. ${ }^{25}$ The use of the term thymoma is restricted to thymic epithelial tumours with minimal or no atypia; those displaying obvious histological and clinical malig-

Table 3 Significance (p) of correlation between five, 10, 15, and 20 year survival and histological type of thymoma

\begin{tabular}{lrrrr}
\hline $\begin{array}{l}\text { Histological } \\
\text { types }\end{array}$ & $5 y$ & $10 y$ & $15 y$ & $20 y$ \\
\hline CT $v$ MT & $<<0.001$ & $<<0.001$ & $<<0.001$ & $<<0.001$ \\
MT $v$ MxT & 0.016 & 0.003 & 0.003 & 0.003 \\
CT $v$ MxT & 0.004 & 0.015 & $0.119^{*}$ & $0.119^{*}$ \\
\hline
\end{tabular}

*Not significant. CT-cortical thymoma; MT-medullary thymoma; MxT - mixed thymoma. 


\section{Survival}

(\%)

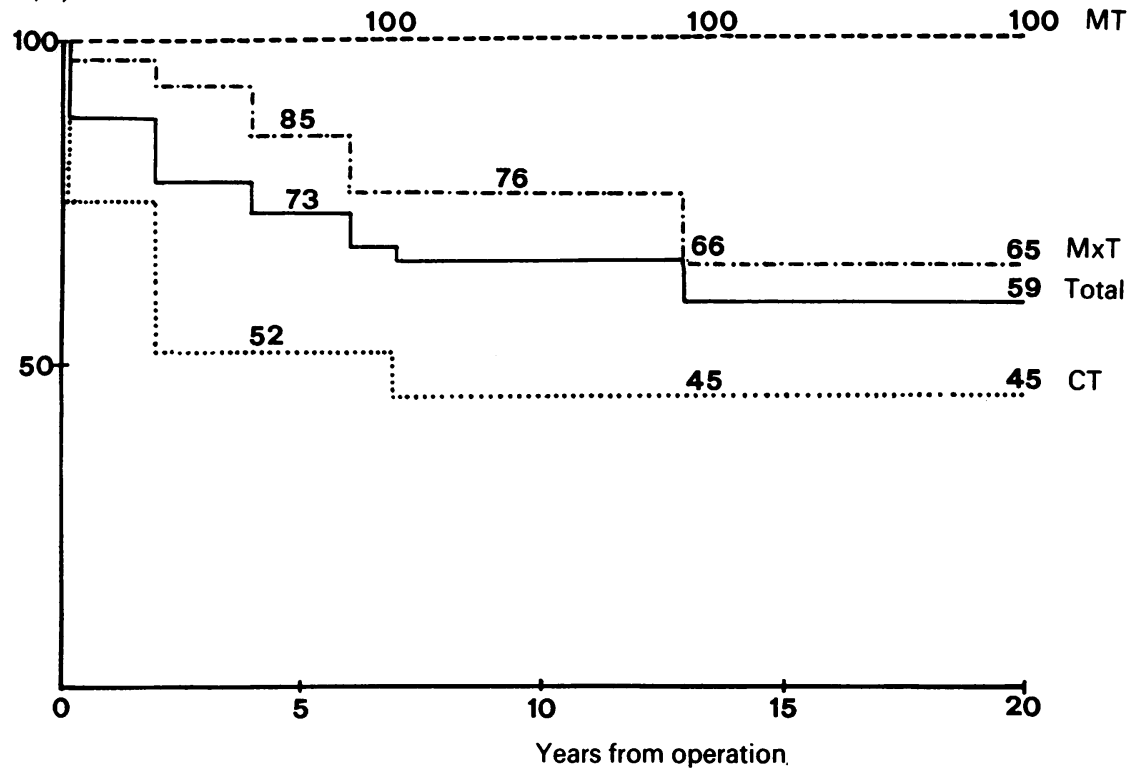

Fig 4 Actuarial survival of patients undergoing operation. $M T$-medullary thymoma; $M x T-$ mixed thymoma; CT-cortical thymoma.

nancy are more appropriately termed thymic carcinoma. ${ }^{10}$ Individual thymomas differ widely in gross morphology and clinical behaviour. Some are apparently benign, well encapsulated lesions, whereas others behave as malignant tumours, with local infiltration and intrathoracic metastases. ${ }^{2341112}$ Histologically, they are generally classified on purely morphological grounds as predominantly lymphocytic, predominantly epithelial, or mixed. ${ }^{235}$ This descriptive approach has little prognostic value as all thymomas are epithelial neoplasms, and lymphoid cells, though often present, are an accessory, non-neoplastic component. Not surprisingly therefore studies based on such classifications ${ }^{1113}$ fail to provide significant clinicopathological correlations.

In 1985 Marino and Müller-Hermelink proposed a new classification based on morphology and immunohistochemistry, ${ }^{614}$ 15 in which a histogenesis based on the different subsets of thymic epithelial cells was suggested. Cortical thymomas were related morphologically and phenotypically to thymic cortical epithelial cells, and medullary thymomas to medullary epithelial cells. Mixed thymomas were also described, and further divided into three subgroups - the mixed common type, mixed with cortical predominance, and mixed with medullary predominance. This subdivision seemed to us difficult to apply and sometimes arbitrary. We therefore classified mixed tumours with cortical predominance with cortical thymomas and those with medullary predominance with medullary thymomas.

Analysis of survival data showed significant differences in prognosis among the three main types. Medullary thymoma is a comparatively rare, benign tumour, arising late in life and usually not associated with myasthenia gravis. It presents as an encapsulated tumour, which grows slowly and does not infiltrate surrounding structures or metastasise. It responds well to surgical resection. In our series, even though postoperative radiotherapy was not given, there were no tumour related deaths or recurrences.

Cortical thymoma, on the other hand, behaves as a malignant tumour. Six of our 30 patients had inoperable disease, five intrathoracic metastases, and five local recurrence requiring further surgery. It usually presents in the middle decades of life as a locally invasive tumour at stage III or IV. Resection should be regarded as the first step in a multidisciplinary approach to treatment including radiotherapy, chemotherapy, or both.

Mixed thymoma is intermediate in its behaviour between medullary and cortical thymoma. These tumours may present as stage I or II disease, but should be considered potentially malignant. Post- 


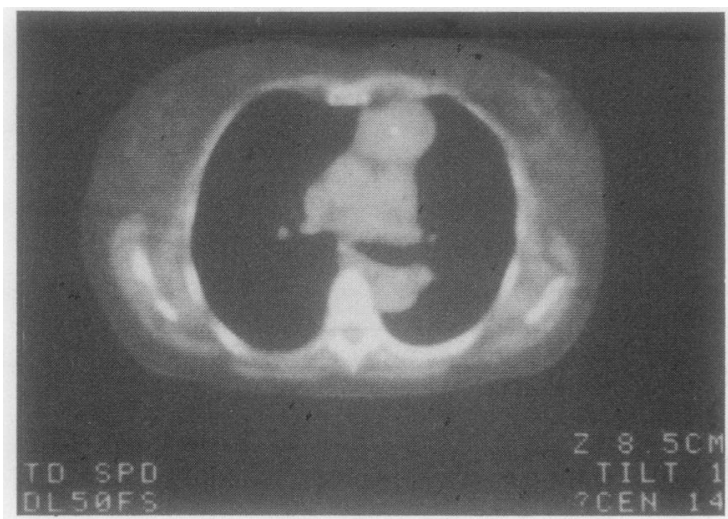

(a)

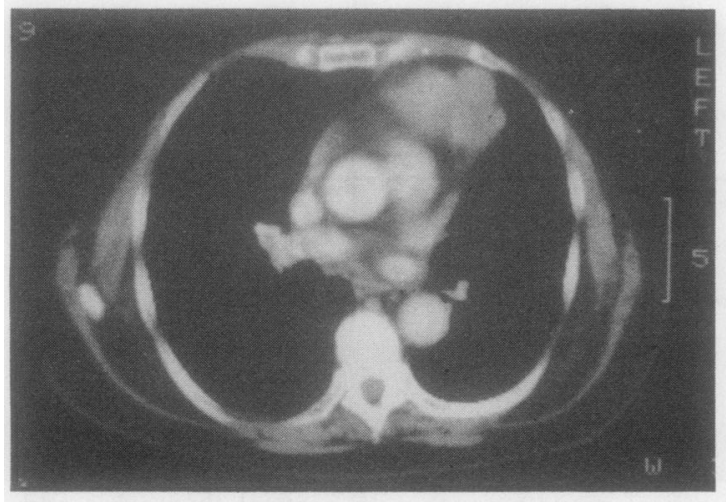

(b)

Fig 5 Computed tomography scans of a medullary tumour in (a) 1981 and (b) 1986, when the tumour has increased in size but clear cleavage planes between it and the mediastinal organs are visible.

operative radiotherapy should always be administered, as tumour related deaths occurred even in patients with stage I disease.

In conclusion, our study of a large series of patients with thymoma shows that medullary, cortical, and mixed tumours differ in both clinical behaviour and prognosis, thus confirming the findings of MüllerHermelink et al.${ }^{14}$ Medullary thymoma is benign, and may be treated by surgery alone. Cortical thymoma must be considered a malignant tumour, and surgical treatment should be performed as the first step of a multidisciplinary approach that includes postoperative radiotherapy in all patients and chemotherapy in selected cases. Mixed thymoma has a better prognosis than cortical thymoma, but the treatment must be that of a potentially malignant tumour and surgical resection must be followed by radiotherapy even in stage I or II lesions.

\section{References}

1 Levine GD, Rosai J. Thymic hyperplasia and neoplasia: a review of current concepts. Hum Pathol 1978;9: 495-515.

2 Rosai J, Levine GD. Tumors of the thymus. In: Atlas of tumor pathology. 2nd series, fascicle 13. Washington DC: Armed Forces Institute of Pathology, 1976.

3 Bernatz PE, Harrison EG, Clagett OT. Thymoma: Clinicopathological study. J Thorac Cardiovasc Surg 1961;42:424-44.

4 Salyer WR, Eggleston JC. Thymoma: a clinical and pathological study of 65 cases. Cancer 1976;37:229-49.

5 Lattes R. Thymoma and other tumors of the thymus: an analysis of 107 cases. Cancer 1962;15:1224-60.

6 Marino M, Müller-Hermelink HK. Thymoma and thymic carcinoma. Relation of thymoma epithelial cells to the cortical and medullary differentiation of the thymus. Virchows Arch 1985;407:119-49.

7 Rendina EA, Pescarmona EO, Venuta F, et al. Thymoma: a clinico-pathologic study based on newly developed morphologic criteria. Tumori 1988;74: 79-84.

8 Masaoka A, Monden Y, Nakahara K, Tanaka T. Follow-up study of thymomas with references to their clinical stages. Cancer 1981;48:2485-92.

9 Kaplan EL, Meier P. Nonparametric estimation from incomplete observations. American Statistical Association Journal 1958;53:457-80.

10 Snover DC, Levine GD, Rosai J. Thymic carcinoma: five distinctive histological variants. Am J Surg Pathol 1982;6:451-70.

11 Batata MA, Martini N, Huvos AG, Aguilar RI, Beattie EJ. Thymomas: Clinicopathologic features, therapy and prognosis. Cancer 1974;34:389-96.

12 Lattes R, Jonas S. The pathological and clinical features in 80 cases of thymoma. Bull NY Acad Med 1957; 33:154-7.

13 Maggi G, Giaccone G, Donadio M, et al. Thymomas. A review of 169 cases, with particular reference to results of surgical treatment. Cancer 1985;58:765-76.

14 Müller-Hermelink HK, Marino M, Palestro G. Pathology of thymic epithelial tumours. In: MüllerHermelink HK, ed. The human thymus. Berlin: Springer-Verlag, 1986. (Current problems in pathology, No 75.)

15 Müller-Hermelink HK, Marino M, Palestro G, Schumcher $U$, Kirchner $T$. Immunohistological evidences of cortical and medullary differentiation in thymoma. Virchows Arch 1985;408:143-61. 